\title{
Urinary F2-Isoprostanes and Metabolic Markers of Fat Oxidation
}

\author{
Dora Il'yasova, ${ }^{1}$ Lynne E. Wagenknecht, ${ }^{2}$ Ivan Spasojevic, ${ }^{3}$ Steven Watkins, ${ }^{4}$ \\ Donald Bowden, ${ }^{5}$ Frances Wang, ${ }^{3}$ and Ralph B. D'Agostino Jr. ${ }^{2}$ \\ ${ }^{1}$ School of Public Health, Georgia State University, 140 Decatur Street, Urban Life Building, Atlanta, GA 30303, USA \\ ${ }^{2}$ Wake Forest School of Medicine, Public Health Sciences, Winston-Salem, NC 27157, USA \\ ${ }^{3}$ Duke Cancer Institute, Duke University Medical Center, 2424 Erwin Road, Durham, NC 27705, USA \\ ${ }^{4}$ Lipomics Technologies, Division of Metabolon, 3410 Industrial Boulevard, West Sacramento, CA 95691, USA \\ ${ }^{5}$ Center for Genomics and Personalized Medicine Research, Wake Forest School of Medicine, Winston-Salem, NC 27157, USA
}

Correspondence should be addressed to Dora Il'yasova; dilyasova@gsu.edu

Received 18 December 2014; Revised 19 January 2015; Accepted 25 January 2015

Academic Editor: Janusz Gebicki

Copyright (c) 2015 Dora Il'yasova et al. This is an open access article distributed under the Creative Commons Attribution License, which permits unrestricted use, distribution, and reproduction in any medium, provided the original work is properly cited.

\begin{abstract}
Metabolomic studies of increased fat oxidation showed increase in circulating acylcarnitines C2, C8, C10, and C12 and decrease in C3, C4, and C5. We hypothesize that urinary F2-isoprostanes reflect intensity of fatty acid oxidation and are associated with circulating C2, C8, C10, and C12 directly and with C3, C4, and C5 inversely. Four urinary F2-isoprostane isomers and serum acylcarnitines are quantified using LC-MS/MS within the Insulin Resistance Atherosclerosis Study nondiabetic cohort $(n=$ 682). Cross-sectional associations between fasting urinary F2-isoprostanes (summarized as a composite index) and the selected acylcarnitines are examined using generalized linear models. F2-isoprostane index is associated with C2 and C12 directly and with C5 inversely: the adjusted beta coefficients are $0.109,0.072$, and -0.094 , respectively $(P<0.05)$. For these acylcarnitines and for F2isoprostanes, the adjusted odds ratios (ORs) of incident diabetes are calculated from logistic regression models: the ORs (95\% CI) are 0.77 (0.60-0.97), 0.79 (0.62-1.01), 1.18 (0.92-1.53), and 0.51 (0.35-0.76) for C2, C12, C5, and F2-isoprostanes, respectively. The direction of the associations between urinary F2-isoprostanes and three acylcarnitines (C2, C5, and C12) supports our hypothesis. The inverse associations of $\mathrm{C} 2$ and $\mathrm{C} 12$ and with incident diabetes are consistent with the suggested protective role of efficient fat oxidation.
\end{abstract}

\section{Introduction}

Urinary F2-isoprostanes are validated indices of human oxidative status, reflecting an overall generation of free radicals, among them reactive oxygen species (ROS) [13]. Commonly, any statistically significant elevation of systemic F2-isoprostanes (by any amount) is interpreted as harmful oxidative stress [1]. A prominent example is the conventional view that the elevated F2-isoprostane levels in obesity represent obesity-induced oxidative stress and a mechanistic link between obesity and cardiovascular risk [4]. In contrast, our research found that elevated urinary F2isoprostanes levels present a favorable trait predicting lower risks of weight gain and type 2 diabetes $[5,6]$; and the findings on the risk of weight gain have been independently confirmed in another cohort [7]. To reconcile the two aspects of urinary F2-isoprostanes, that is, as favorable metabolic trait and as systemic indices of ROS, we hypothesized that mitochondrial oxidative metabolism is the main determinant of urinary F2-isoprostane levels [8], because mitochondria are the major endogenous source of ROS [9]. We also hypothesized that fatty acid oxidation plays the predominant role in the connection between mitochondrial metabolism and urinary F2-isoprostane levels, because mitochondrial fatty acid oxidation (a) produces higher levels of ROS [10] and (b) largely accounts for the peripheral tissue oxidative metabolism [11]. This hypothesis builds upon the protective roles that the intensive mitochondrial metabolism, fatty acid 
oxidation, and physical activity play against obesity and type 2 diabetes [12] and the well-known fact that physical activity induces increases in F2-isoprostane levels $[13,14]$.

In earlier publications, we examined several epidemiological evidences supporting the hypothesized connection between urinary F2-isoprostanes and fat oxidation. We found that a racial group with lower levels of fat oxidation, that is, African Americans [15], congruently has lower levels of urinary F2-isoprostanes [16]. We also demonstrated that fasting levels of nonesterified fatty acids, which are known to stimulate muscle fat oxidation, correlate directly with urinary F2-isoprostanes [17]. Further exploration of our hypothesis has become possible due to the recent metabolomic studies of circulating metabolites in conditions with intensified fat oxidation, namely, during fasting and moderate intensity exercise $[18,19]$. Specifically, the metabolomic study of fasting demonstrated that circulating levels of C3 (propionylcarnitine), C4 (butyrylcarnitine), and C5 (valerylcarnitine), all derivatives of amino acid oxidation, decline, whereas C2 (acetylcarnitine) levels increase [18]. The study of moderate intensity exercise found that a 60-120 min run resulted in a transient increase of C8 (octanoylcarnitine), C10 (decanoylcarnitine), and C12 (dodecanoylcarnitine) levels [19]. If urinary F2-isoprostanes correlate with the intensity of fat oxidation, it is logical to expect that these biomarkers are associated with $\mathrm{C} 2, \mathrm{C} 8, \mathrm{C} 10$, and $\mathrm{C} 12$ directly and with C3, C4, and C5 inversely. We also hypothesized that these same acylcarnitines are associated with incident type 2 diabetes congruently with the inverse association between F2-isoprostanes and type 2 diabetes [6]. We explored these questions by examining both F2-isoprostane and acylcarnitine data from the Insulin Resistance Atherosclerosis Study (IRAS) multiethnic cohort.

\section{Research Design and Methods}

2.1. Study Population. The IRAS is a well-characterized multiethnic cohort described in detail [17]. The analytical cohort includes the baseline nondiabetic participants with normal (NGT) or impaired glucose tolerance (IGT) only, with available measurements of urinary F2-isoprostanes and acylcarnitines ( $n=682$ ), among them 114 participants developing type 2 diabetes during the follow-up.

2.2. Measurements. All subjects fasted for 12 hours and refrained from heavy exercise, smoking, and alcohol consumption for 24 hours before the visit. The metabolic and anthropometric measurements were described earlier [17]. Participants had a frequently sampled glucose tolerance test for which insulin sensitivity $\left(S_{\mathrm{I}}\right)$ and acute insulin response (AIR) were calculated. Four isomers of F2-isoprostanes-iPF2 $\alpha$-III, 2,3-dinor-iPF2 $\alpha$-III, iPF2 $\alpha$-VI, and 8,12 -iso-iPF2 $\alpha$-VI-are quantified in morning spot urine samples (stored at $-70^{\circ} \mathrm{C}$ ) by liquid chromatography with tandem mass spectrometry detection (LC-MS/MS) as previously described. F2-isoprostane levels are corrected by urinary creatinine to account for differences in urine dilution [3].
Acylcarnitines are also measured by LC-MS/MS. Deuterium-labeled internal standards were added to 25 microliters of serum and the mixture was solubilized in methanol followed by a crash extraction and then injected onto an Atlantis HILIC Column connected to a Waters Xevo triple quadrupole mass spectrometer (Waters, MA). Acylcarnitines were ionized via positive electrospray and the mass spectrometer was operated in the tandem MS mode. The absolute concentration of each acylcarnitine is determined by comparing the corresponding peak to that of the relevant internal standard.

2.3. Statistical Analysis. Spearman correlation coefficient is used to examine crude correlations between acylcarnitines. For F2-isoprostanes, we calculated a composite index that ranks individuals based on all four measurements: $\left[\left(\mathrm{X}_{i}-\right.\right.$ $\mathrm{M} 1) / \mathrm{SD} 1+\left(\mathrm{X} 2_{i}-\mathrm{M} 2\right) / \mathrm{SD} 2+\left(\mathrm{X} 3_{i}-\mathrm{M} 3\right) / \mathrm{SD} 3+\left(\mathrm{X} 4_{i}-\right.$ $\mathrm{M} 4) / \mathrm{SD} 4] / 4$, where " $i$ " is a notation for a participant; values of four F2-isoprostanes species (X1-4) were standardised by subtracting estimated mean (M1-4) and divided by standard deviation (SD1-4). For acylcarnitines, we use natural-log transformed variables to reduce the influence of high values.

Adjusted beta coefficients for the associations between F2-isoprostane index and the acylcarnitines are calculated from generalized linear models. The minimally adjusted models include demographic variables (age, gender, and race/ethnicity) and BMI; the fully adjusted models include additional metabolic variables (IGT, insulin sensitivity $\left[\log \left(S_{\mathrm{I}}+1\right)\right]$, and AIR). The association between the acylcarnitines and incident diabetes $(n=114)$ is quantified by odds ratios calculated from logistic regression models, with the covariates selected by our previously published analysis [6].

\section{Results}

Our study population is metabolically and ethnically diverse, with 43\% Non-Hispanic and 32\% Hispanic Whites and 25\% African Americans and 26\% normal, 44\% overweight, and $29 \%$ obese (Table 1 ). One third of the study population is NGT with two-thirds being IGT. The observed correlations between the examined acylcarnitines are in agreement with the findings from the metabolomics studies [18, 19]. As expected, the strongest correlations are found between $\mathrm{C} 8$, $\mathrm{C} 10$, and $\mathrm{C} 12(0.82 \leq r \leq 0.97, P<0.05)$ and between $\mathrm{C} 3$, C4, and C5 $(0.43 \leq r \leq 0.59, P<0.05)$. C2 correlates most strongly with $\mathrm{C} 12(r=0.41, P<0.05)$ as compared to other acylcarnitines.

Examining the associations between fasting levels of F2isoprostanes (expressed as composite index) and acylcarnitines, we find that beta coefficients are consistently positive for the associations with $\mathrm{C} 2, \mathrm{C} 8, \mathrm{C} 10$, and C12, whereas for $\mathrm{C} 3$, $\mathrm{C} 4$, and $\mathrm{C} 5$ the beta coefficients are negative (Table 2). Three acylcarnitines show statistically significant associations: $P$ values for the beta coefficients are 0.03 for C2, C5, and C12. The magnitude of the beta coefficients and the standard errors are generally consistent between the minimally and the fully adjusted models (Table 2). For example, beta coefficients for 
TABLE 1: Characteristics of the study population $(n=682)$.

\begin{tabular}{|c|c|}
\hline Characteristics & $\begin{array}{c}\text { Mean (SD) or } \\
\text { number (\%) }\end{array}$ \\
\hline Age (years) & $54.5(8.4)$ \\
\hline Sex, female (number, \%) & $385(56.5)$ \\
\hline \multicolumn{2}{|l|}{ Ethnicity (number, \%) } \\
\hline Non-Hispanic White & $291(43)$ \\
\hline African American & $174(25)$ \\
\hline Hispanic White & $217(32)$ \\
\hline Normal glucose tolerance, NGT (number, \%) & $226(33)$ \\
\hline Impaired glucose tolerance IGT (number, \%) & $456(67)$ \\
\hline \multicolumn{2}{|l|}{ BMI $\left(\mathrm{kg} / \mathrm{m}^{2}\right)$} \\
\hline Normal $(<25)$ & $179(26)$ \\
\hline Overweight (25-29.9) & $303(44)$ \\
\hline Obese $(\geq 30)$ & $199(29)$ \\
\hline Missing & 1 \\
\hline iPF $2 \alpha$-III (ng/mg creatinine) & $0.25(0.20)$ \\
\hline 2,3-dinor-iPF2 $\alpha$-III (ng/mg creatinine) & $4.32(3.05)$ \\
\hline iPF $2 \alpha$-VI (ng/mg creatinine) & $6.46(4.08)$ \\
\hline 8,12 -iso-iPF $2 \alpha$-VI (ng/mg creatinine) & $4.19(2.92)$ \\
\hline F2-isoprostane composite index & $1.41(0.80)$ \\
\hline Acetylcarnitine, C2 (nmole/g) & $7.16(2.45)$ \\
\hline Propionylcarnitine, C3 (nmole/g) & $0.37(0.12)$ \\
\hline Butyrylcarnitine, C4 (nmole/g) & $0.19(0.11)$ \\
\hline Valerylcarnitine, C5 (nmole/g) & $0.15(0.05)$ \\
\hline Octanoylcarnitine, C8 (nmole/g) & $0.17(0.12)$ \\
\hline Decanoylcarnitine, C10 (nmole/g) & $0.33(0.29)$ \\
\hline Dodecanoylcarnitine, C12 (nmole/g) & $0.21(0.11)$ \\
\hline
\end{tabular}

Mean (SD) presented for continuous variables; F2-isoprostane composite index was calculated using all four F2-isoprostane measurements as follows: each value was standardized (divided by the standard deviation) and mean of the four standardized values was calculated for each participant.

the association between F2-isoprostanes and C2 correspond to $9.8 \%$ and $10.9 \%$ increase of mean $\mathrm{F} 2$-isoprostane index per standard deviation of C2 distribution.

The three acylcarnitines that are significantly associated with F2-isoprostanes (C2, C5, and C12) are examined as predictors of incident diabetes (Table 3). C2 and $\mathrm{C} 12$ are inversely associated with type 2 diabetes risk ( $P$ values 0.03 and 0.06 , resp.); and $\mathrm{C} 3$ shows a nonsignificant positive association ( $P$ value 0.19 ). The estimated magnitudes of the associations with $\mathrm{C} 2$ and $\mathrm{C} 12$ are very close (odds ratios are 0.77 and 0.79 , resp.). As expected, the association with the composite index of F2-isoprostanes confirms the inverse relationships with type 2 diabetes risk in this analytical cohort (Table 3).

\section{Discussion}

This analysis is inspired by the recent metabolomic studies that examined changes in circulating metabolites within the physiological conditions incurring increased fat oxidation
$[18,19]$. We hypothesized that urinary F2-isoprostane levels reflect a greater ability to oxidize fat and therefore are associated directly with $\mathrm{C} 2, \mathrm{C} 8, \mathrm{C} 10$, and $\mathrm{C} 12$ and inversely with $\mathrm{C} 3, \mathrm{C} 4$, and $\mathrm{C} 5$. The detected trends are consistent with our hypotheses. Significant associations are found for urinary F2-isoprostanes C2, C12, and C5. Acylcarnitine C5 is a metabolite related to catabolism of branched chain amino acids (BCAAs), which is thought to be negatively regulated by mitochondrial fatty acid oxidation [20]. In fact, the metabolomic study of fasting showed an increase in circulating levels of BCAAs, which could result from a decreased catabolism of BCAAs in the condition of increased fat oxidation [18]. Acylcarnitine C2, while potentially derived from multiple fuel sources that generate acetyl-CoA, is known as a metabolite increased by exercise and fasting due in large part to increased fatty acid oxidation [21, 22]. Importantly, the detected associations between F2-isoprostane and acylcarnitines are stable, as shown by the minimally and fully adjusted models in Table 2 . This in conjunction with no findings contradicting our hypothesis insures the consistency of these results.

Additional support for our hypothesis comes from the prospective analysis of type 2 diabetes risk. Our results indicate a trend of inverse associations of $\mathrm{C} 2$ and $\mathrm{C} 12$ with incident type 2 diabetes, whereas C5 shows an opposite trend. These findings are in agreement with the expectations based on the results in Table 2, demonstrating the overall consistency of our results. They also support the suggested protective role of efficient fat oxidation in etiology of type 2 diabetes. Importantly, this analysis utilizes the data from the well-characterized multiethnic cohort, which provides an opportunity to adjust the results for potential confounders and assumes generalizability. In summary, our results provide additional support to the hypothesis that urinary levels of F2isoprostanes are related to the intensity of fatty acid oxidation.

\section{Abbreviations}

$\begin{array}{ll}\text { AIR: } & \text { Acute insulin response } \\ \text { BMI: } & \text { Body mass index } \\ \text { C2: } & \text { Acetylcarnitine } \\ \text { C3: } & \text { Propionylcarnitine } \\ \text { C4: } & \text { Butyrylcarnitine } \\ \text { C5: } & \text { Valerylcarnitine } \\ \text { C8: } & \text { Octanoylcarnitine } \\ \text { C10: } & \text { Decanoylcarnitine } \\ \text { C12: } & \text { Dodecanoylcarnitine } \\ \text { CI: } & \text { Confidence interval } \\ \text { IGT: } & \text { Impaired glucose tolerance } \\ \text { IRAS: } & \text { Insulin Resistance Atherosclerosis Study } \\ \text { NGT: } & \text { Normal glucose tolerance } \\ \text { LC-MS/MS: } & \text { Liquid chromatography with tandem } \\ & \text { mass spectrometry } \\ \text { OR: } & \text { Odds ratio } \\ S_{I}: & \text { Insulin sensitivity. }\end{array}$

\section{Conflict of Interests}

The authors do not have any conflict of interests to declare. 
TABLE 2: Association between F2-isoprostane index and selected acylcarnitines $(n=682)$.

\begin{tabular}{|c|c|c|}
\hline \multirow{2}{*}{ Acylcarnitines } & \multicolumn{2}{|c|}{ Beta coefficient (95\% CI) } \\
\hline & Minimally adjusted model & Fully adjusted model \\
\hline Acetylcarnitine, $\mathrm{C} 2$ & $0.098(0.042,0.154)$ & $0.109(0.051,0.167)$ \\
\hline Propionylcarnitine, C3 & $-0.027(-0.089,0.034)$ & $-0.024(-0.088,0.040)$ \\
\hline Butyrylcarnitine, C4 & $-0.010(-0.067,0.048)$ & $-0.010(-0.069,0.049)$ \\
\hline Valerylcarnitine, C5 & $-0.093(-0.156,-0.030)$ & $-0.094(-0.159,-0.029)$ \\
\hline Octanoylcarnitine, C8 & $0.010(-0.046,0.066)$ & $0.014(-0.044,0.073)$ \\
\hline Decanoylcarnitine, $\mathrm{C} 10$ & $0.016(-0.040,0.071)$ & $0.020(-0.039,0.079)$ \\
\hline Dodecanoylcarnitine, C12 & $0.065(0.009,0.122)$ & $0.072(0.013,0.132)$ \\
\hline
\end{tabular}

Beta coefficients show differences in F2-isoprostanes index associated with a change in acylcarnitines (log-transformed) equal to standard deviation. Minimally adjusted models included the demographic variables (age, gender, and race/ethnicity) and BMI; fully adjusted models included additional metabolic variables (IGT-status, insulin sensitivity, and AIR). Statistically significant results $(P<0.05)$ are in bold.

TABLE 3: Association of the baseline F2-isoprostane index and three selected acylcarnitines with incident type 2 diabetes (114 cases).

\begin{tabular}{lc}
\hline & OR $(95 \%$ CI $)$ \\
\hline F2-isoprostane composite index & $0.51(0.35,0.76)$ \\
Acetylcarnitine, C2 & $0.77(0.60,0.97)$ \\
Valerylcarnitine, C5 & $1.18(0.92,1.53)$ \\
Dodecanoylcarnitine, C12 & $0.79(0.62,1.01)$ \\
\hline
\end{tabular}

ORs adjusted for age, gender, ethnicity, clinic and baseline BMI, and IGT-status and scaled to SD of F2-isoprostanes or acylcarnitines (logtransformed).

\section{Acknowledgments}

The authors thank Dr. Sean Adams, the USDA Agricultural Research Service Western Human Nutrition Research Center, for his help in the interpretation of the results. This study was supported by the National Institutes of Health Grant 1R01DK081028.

\section{References}

[1] S. Basu, "F2-isoprostanes in human health and diseases: from molecular mechanisms to clinical implications," Antioxidants and Redox Signaling, vol. 10, no. 8, pp. 1405-1434, 2008.

[2] D. Il'yasova, P. Scarbrough, and I. Spasojevic, "Urinary biomarkers of oxidative status," Clinica Chimica Acta, vol. 413, no. 19-20, pp. 1446-1453, 2012.

[3] D. Il'Yasova, I. Spasojevic, F. Wang et al., "Urinary biomarkers of oxidative status in a clinical model of oxidative assault," Cancer Epidemiology Biomarkers and Prevention, vol. 19, no. 6, pp. 1506-1510, 2010.

[4] H. Otani, "Oxidative stress as pathogenesis of cardiovascular risk associated with metabolic syndrome," Antioxidants and Redox Signaling, vol. 15, no. 7, pp. 1911-1926, 2011.

[5] D. Il'Yasova, F. Wang, I. Spasojevic, K. Base, R. B. D’Agostino Jr., and L. E. Wagenknecht, "Urinary F 2-isoprostanes, obesity, and weight gain in the IRAS cohort," Obesity, vol. 20, no. 9, pp. 1915-1921, 2012.

[6] D. Il'yasova, I. Spasojevic, K. Base et al., "Urinary f2isoprostanes as a biomarker of reduced risk of type 2 diabetes," Diabetes Care, vol. 35, no. 1, pp. 173-174, 2012.
[7] A. M. Kanaya, C. L. Wassel, P. J. Stoddard et al., "F2-isoprostanes and adiposity in older adults," Obesity, vol. 19, no. 4, pp. 861-867, 2011.

[8] D. Ilyasova, J. D. Morrow, and L. E. Wagenknecht, "Urinary F 2-isoprostanes are not associated with increased risk of type 2 diabetes," Obesity Research, vol. 13, no. 9, pp. 1638-1644, 2005.

[9] T. R. Figueira, M. H. Barros, A. A. Camargo et al., "Mitochondria as a source of reactive oxygen and nitrogen species: from molecular mechanisms to human health," Antioxidants and Redox Signaling, vol. 18, no. 16, pp. 2029-2074, 2013.

[10] C. L. Quinlan, I. V. Perevoshchikova, M. Hey-Mogensen, A. L. Orr, and M. D. Brand, "Sites of reactive oxygen species generation by mitochondria oxidizing different substrates," Redox Biology, vol. 1, no. 1, pp. 304-312, 2013.

[11] R. A. Jackson, J. B. Hamling, P. M. Blix, and J. D. N. Nabarro, "Relationship among peripheral glucose uptake, oxygen consumption, and glucose turnover in postabsorptive man," The Journal of Clinical Endocrinology \& Metabolism, vol. 59, no. 5, pp. 857-860, 1984.

[12] L. Heilbronn, S. R. Smith, and E. Ravussin, "Failure of fat cell proliferation, mitochondrial function and fat oxidation results in ectopic fat storage, insulin resistance and type II diabetes mellitus," International Journal of Obesity, vol. 28, supplement 4, pp. S12-S21, 2004.

[13] K. Margonis, I. G. Fatouros, A. Z. Jamurtas et al., "Oxidative stress biomarkers responses to physical overtraining: implications for diagnosis," Free Radical Biology and Medicine, vol. 43, no. 6, pp. 901-910, 2007.

[14] L. W. Jones, N. D. Eves, I. Spasojevic, F. Wang, and D. Il'yasova, "Effects of aerobic training on oxidative status in postsurgical non-small cell lung cancer patients: a pilot study," Lung Cancer, vol. 72, no. 1, pp. 46-51, 2011.

[15] C. Weyer, S. Snitker, C. Bogardus, and E. Ravussin, "Energy metabolism in African Americans: potential risk factors for obesity," The American Journal of Clinical Nutrition, vol. 70, no. 1, pp. 13-20, 1999.

[16] D. Il'Yasova, F. Wang, I. Spasojevic, K. Base, R. B. D’Agostino Jr., and L. E. Wagenknecht, "Racial differences in urinary F2-isoprostane levels and the cross-sectional association with BMI," Obesity (Silver Spring), vol. 20, no. 10, pp. 2147-2150, 2012.

[17] D. Il'yasova, F. Wang, R. B. D’Agostino Jr., A. Hanley, and L. E. Wagenknecht, "Prospective association between fasting NEFA and type 2 diabetes: impact of post-load glucose," Diabetologia, vol. 53, no. 5, pp. 866-874, 2010. 
[18] S. Krug, G. Kastenmüller, F. Stückler et al., "The dynamic range of the human metabolome revealed by challenges," The FASEB Journal, vol. 26, no. 6, pp. 2607-2619, 2012.

[19] R. Lehmann, X. Zhao, C. Weigert et al., "Medium chain acylcarnitines dominate the metabolite pattern in humans under moderate intensity exercise and support lipid oxidation," PLoS ONE, vol. 5, no. 7, Article ID el1519, 2010.

[20] S. H. Adams, "Emerging perspectives on essential amino acid metabolism in obesity and the insulin-resistant state," Advances in Nutrition, vol. 2, no. 6, pp. 445-456, 2011.

[21] F. B. Stephens, D. Constantin-teodosiu, and P. L. Greenhaff, "New insights concerning the role of carnitine in the regulation of fuel metabolism in skeletal muscle," The Journal of Physiology, vol. 581, no. 2, pp. 431-444, 2007.

[22] C. L. Hoppel and S. M. Genuth, "Urinary excretion of acetylcarnitine during human diabetic and fasting ketosis.," The American Journal of Physiology, vol. 243, no. 2, pp. E168-E172, 1982. 


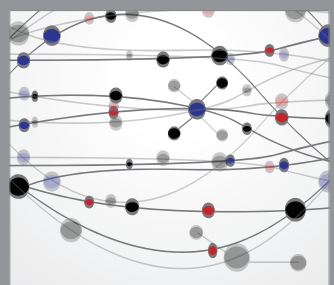

The Scientific World Journal
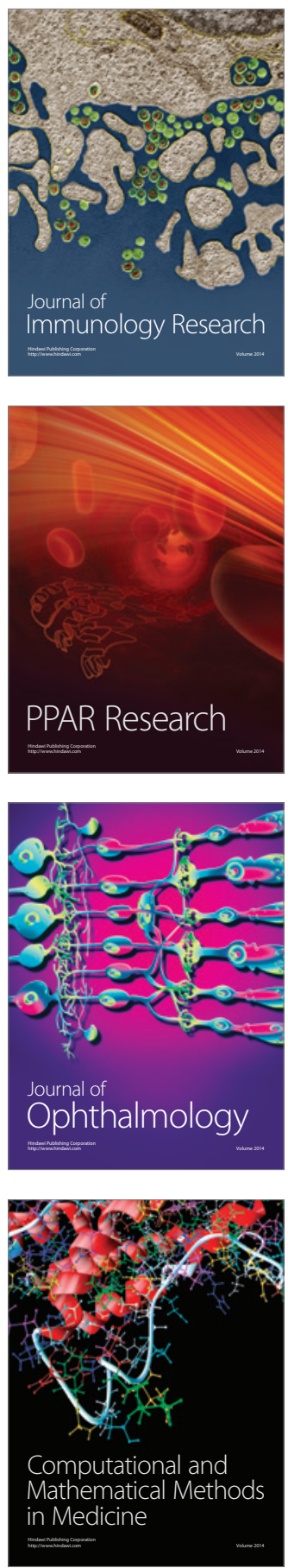

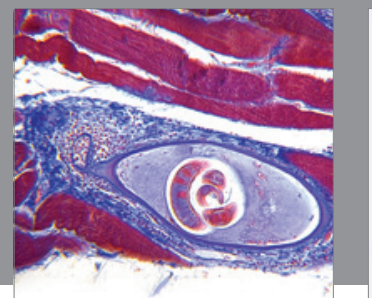

Gastroenterology

Research and Practice
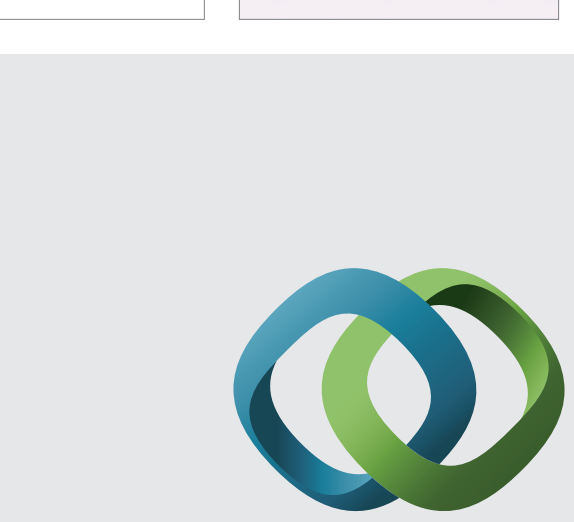

\section{Hindawi}

Submit your manuscripts at

http://www.hindawi.com
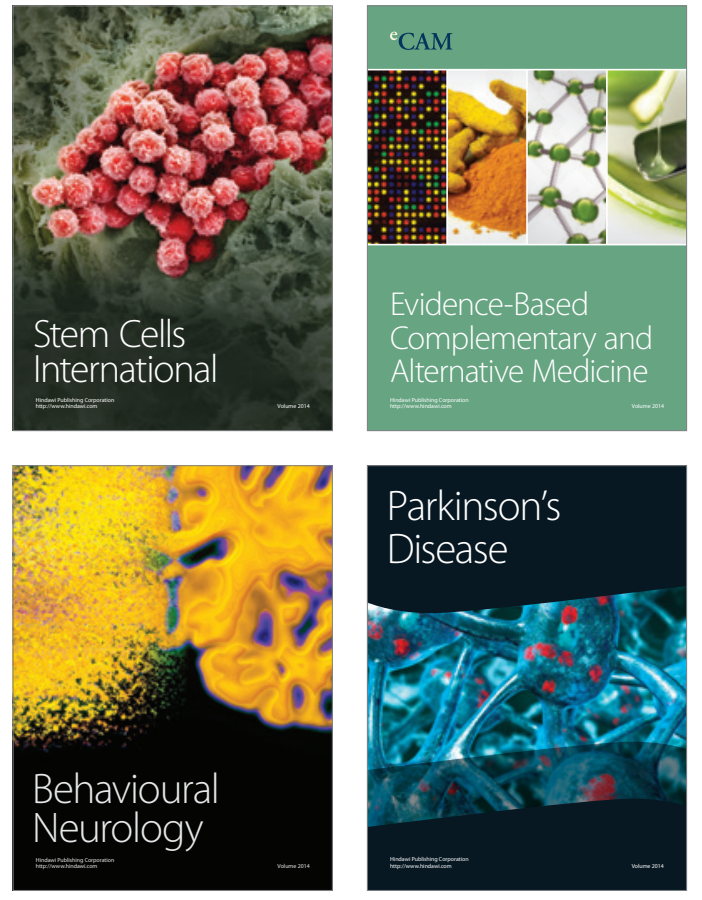
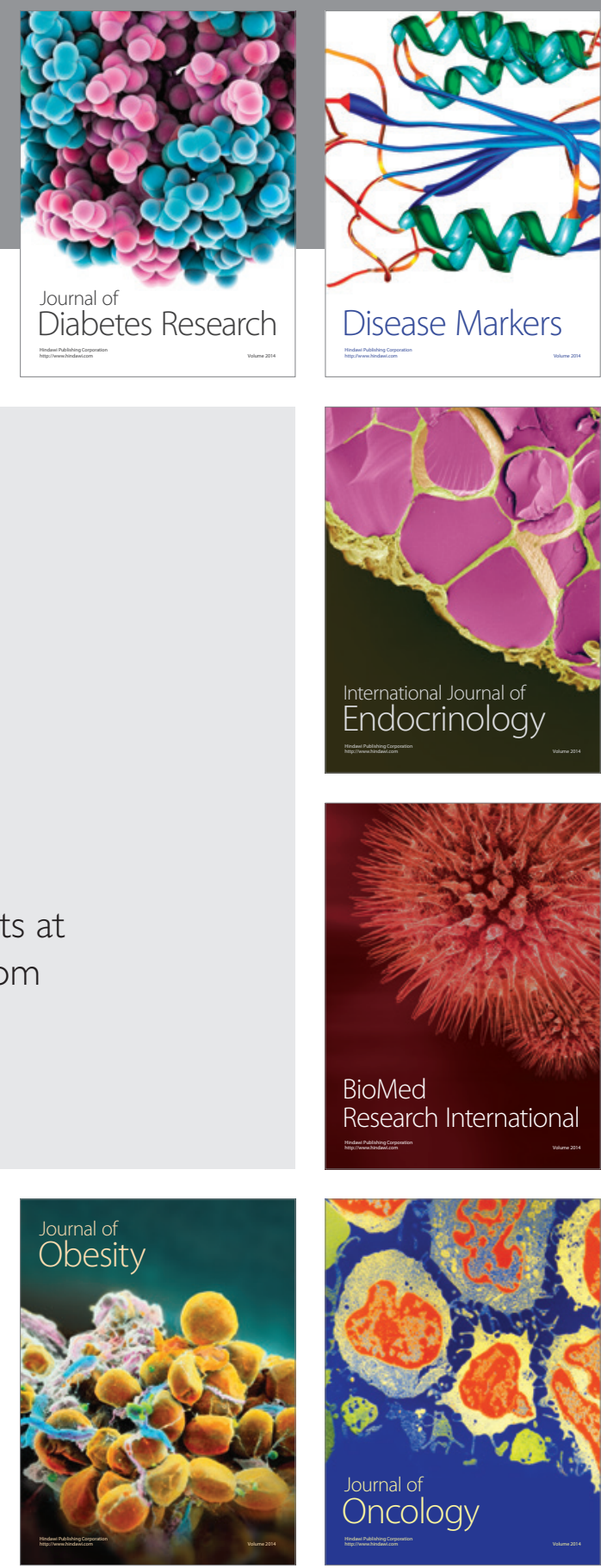

Disease Markers
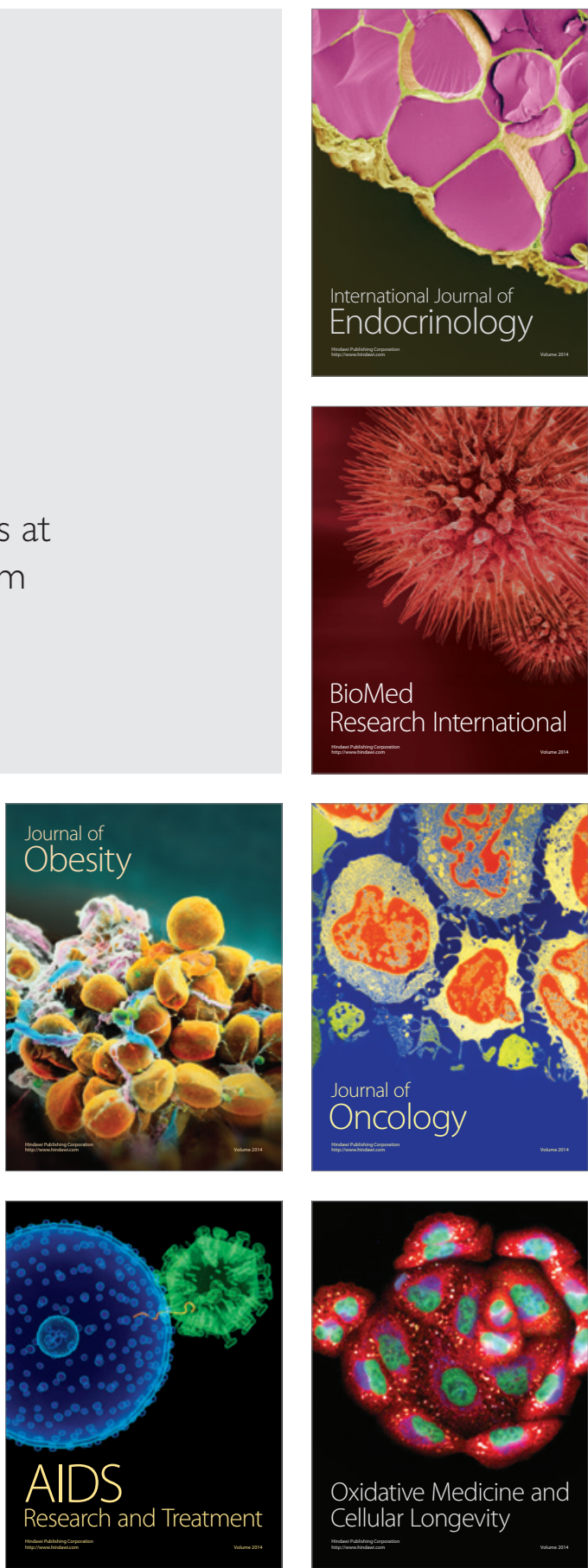